Nuclear Physics B3̈50 (1991) 563-588

North-Holland

\title{
DOMAIN WALLS, SURFACE TENSION AND WETTING IN THE THREE-DIMENSIONAL THREE-STATE POTTS MODEL
}

\author{
F. KARSCH AND A. PATKÓS* \\ CERN, Theory Ditision, 1211 Geneta 23, Switzerland \\ Received 7 May 1990 \\ (Revised 6 July 1990)
}

\begin{abstract}
We study the thermodynamic properties of interfaces between differently oriented ordered domains in the three-dimensional three-state Potts model. We perform simulations on lattices with cylindric geometry, using parallel and rotated fixed boundary conditions. Systematic control over finite-size effects and the number of interfaces is achieved. Global and local characterization of the interfacial structure is given and substantial evidence for complete wetting is presented.
\end{abstract}

\section{Introduction}

During recent years the phase structure of QCD at finite temperature has been studied in detail, using Monte Carlo simulation techniques for the lattice regularized theory. There are strong indications that the chiral phase transition in QCD as well as the deconfinement transition in the SU(3) gauge theory are first order. In that case there exists a mixed phase at a critical temperature $T_{c}$ at which bubbles of hadronic phase and quark-gluon plasma phase can coexist. This has applications in the discussion of the phase transition in the early universe [1-3] as well as in the heavy-ion physics $[4,5]$. In this context it is of particular interest to achieve a quantitative understanding of the dynamics of interfaces between the hadronic and quark-gluon plasma phase.

There have recently been attempts to calculate the surface tension in QCD, i.e. in the pure SU(3) gauge sector, on the lattice [6-8]. The main problem in such a calculation is to stabilize the interface between the two phases at the critical temperature. So far this has been achieved by introducing a temperature gradient in the system, which forces it into different phases on opposite sides of the interface. This approach has also been tested in simpler two-dimensional Potts models [9-11]. The determination of the interface tension then involves an extrapolation to zero temperature gradient. An alternative approach, which we are

\footnotetext{
* On leave from Department of Atomic Physics, Eötvös University, Budapest, Hungary.
} 
going to analyze here, is to enforce the appearance of an interface through the choice of boundary conditions. This is a natural approach in mean field calculations [12,13]. In a Monte Carlo simulation, however, one faces the problem that the number of interfaces and their location is entirely controlled by the dynamics of the system.

The deconfinement phase transition in SU(3) gauge theory is driven by the spontaneous breaking of a global $Z(3)$ symmetry. It is expected that the dynamics of the transition is closely related to the corresponding $Z(3)$ symmetry-breaking transition in three-dimensional spin models [14]. In fact, there is numerical evidence that the effective action describing the SU(3) deconfinement phase transition is a three-dimensional spin model with ferromagnetic, short-ranged interactions [15]. Its properties are closely related to that of the ferromagnetic three-dimensional, three-state Potts model. Because of this relation to QCD the Potts model has recently been studied in great detail [16-18]. In particular, the finite-size scaling behavior of thermodynamic quantities gave additional support to the existence of a first-order phase transition in this model.

The surface tension for the order-disorder interface in the Potts model, which corresponds to the interface between a hadronic and a quark-gluon plasma phase in QCD, has been studied recently [19] using the approach of refs. [6, 7]. Quantitative results for the surface tension in the Potts model with ferromagnetic nearest neighbour coupliings are, of course, not directly applicable to QCD. It is, however, conceivable that a semi-quantitative study of the interface dynamics could be performed in the framework of a three-dimensional effective spin model with global Z(3) symmetry. A mapping of the finite-temperature pure gauge QCD onto an effective Potts action with up to 40 two-, three- and four-spin couplings has been performed recently [15] and could be used for such an investigation.

Besides the order-disorder interfaces, corresponding to transitory regions between hadronic and quark-gluon plasma phases in QCD, there exist also interfaces between differently oriented, ordered domains. Unlike the disorder-order interfaces these order-order domain walls exist for all temperatures in the symmetry-broken phase of the Potts model, as well as the SU(3) gauge theory. At the critical temperature the three ordered phases and the disordered phase can coexist, and the stability of the interfaces between them requires the fulfillment of the following inequality [20]:

$$
\alpha_{\text {ord }} \leqslant 2 \alpha_{\text {dis }} \quad\left(\beta=\beta_{c}\right)
$$

Here $\alpha$ denotes the surface tension and the indices dis (ord) stand for the disorder-order (order-order) interfaces. The inverse temperature is denoted as usual by $\beta$. If the equality holds in (1) a disordered domain appears at $\beta_{c}$ spontaneously in form of a "two-dimensional" layer separating two differently magnetized ordered domains without the need of supercooling. This is the phe- 
nomenon of complete wetting, which has prospective interest in particle physics too. If, however, the strict inequality holds at coexistence, the new phase would form droplets (incomplete wetting) and actually it could appear only when a slight supercooling would compensate the extra surface energy necessary for the equilibrium. The realization of complete wetting will also be analyzed in the present paper together with related scaling laws, which are expected to show mean-field behavior [21]. In the case of complete wetting the critical properties of the interface are expected to be universal. Quantities like the magnetization on the interface or its width thus will obey the same scaling laws in pure gauge $Q C D$ as in the three-dimensional three-state Potts model. Of course, quantities related to the bulk first-order transition like the surface tension itself will not be universal. The problem of complete wetting is relatively poorly studied with Monte Carlo techniques in the case of the three-dimensional three-state Potts model [22]; therefore, the investigation is of interest for statistical physics too.

The hamiltonian of the three-state Potts model in three dimensions will be used in the following form:

$$
H=-\sum_{x, e} \delta_{s_{x}, s_{x+e}}
$$

The spin variable $s_{x}$, defined on sites $x$ of the lattice, takes three different values: $s_{x}=0,1$ or 2 ; $e$ stands for the lattice unit vectors. We have studied the thermodynamics of this model on lattices of size $L_{\perp}^{2} \times L_{\|}$with periodic boundary conditions in the transverse $(\perp)$ directions and various fixed boundary conditions in the longitudinal ( II) direction.

This paper is organized as follows. In sect. 2 we will discuss some bulk thermodynamic properties of the Potts model on cylindrical lattices with fixed boundary conditions. We will compare the results with those obtained from simulations on cubic lattices of size $L^{3}$ with periodic boundary conditions and discuss the different finite-size effects. In sect. 3 we describe some global properties of order-order interfaces such as their statistical distribution at finite temperature and the influence of various fixed boundary conditions on this. A general analysis of the surface tension is given in sect. 4. The corresponding numerical results are discussed in sect. 5. The thermodynamics on these fluctuating twodimensional layers between ordered phases, which is expected to show the characteristics of a second-order phase transition, is discussed in sect. 6. Finally we give our conclusions in sect. 7.

\section{Thermodynamics}

It is well known that fixed boundary conditions lead to much stronger finite-size effects than, for instance, periodic boundary conditions. The analysis of the interface tension on such lattices requires a quantitative understanding of these 
effects. In particular, we have to get control over the volume dependence of the critical coupling on lattices with cylindrical geometry and fixed boundary conditions. As the finite-size effects in such a system were not studied in detail before, we start with a discussion of these as a necessary prerequisite for the analysis of the surface tension in sect. 3.

Our simulations have been performed on three-dimensional lattices of size $L_{\perp}^{2} \times L_{\|}$, with transverse sizes $L_{\perp}=20,24$, and 30 , and $L_{\|}$ranging from $3 L_{\perp}$ to $5 L_{1}$. The transverse size was thus about 2-3 times the correlation length determined on cubic lattices in the transition region $\left(\xi_{c} \simeq 10\right)$ [16]. We have concentrated on the coupling region $0.548 \leqslant \beta \leqslant 0.552$, which covers the infinite volume critical coupling [16]

$$
\beta_{\mathrm{c}}=0.55062 \pm 0.00003
$$

as well as the critical region determined on finite cubic lattices. At each $\beta$-value we performed between 300000 and 700000 iterations. We have used lattices with identical, frozen ground-state configurations as boundaries on the first and last planes in the longitudinal direction, i.e.

$$
s\left(x_{\perp}, x_{\|}=1\right)=0, \quad s\left(x_{\perp}, x_{\|}=L_{\|}\right)=0,
$$

and also with different, ground-state configurations, i.e.

$$
s\left(x_{\perp}, x_{\|}=1\right)=0, \quad s\left(x_{\perp}, x_{\|}=L_{\|}\right)=1
$$

In the following we will refer to these as parallel (par.b.c.) and rotated (rot.b.c.) boundary conditions, respectively.

For the analysis of the surface tension using lattices with fixed boundary conditions, it will be essential to have long lattices, $L_{\|} \gg L_{\perp}$, in order to reduce the distortion of the interface (and the thermodynamics) by the boundaries. It will also be important to have a large transverse direction, $L_{\perp} \gg \xi_{c}$, in order to suppress the formation of spurious interfaces. We looked at the volume dependence of thermodynamic quantities in two different limits:

(i) $L_{\|} \rightarrow \infty, L_{\perp}$ fixed;

(ii) $L_{\perp} \rightarrow \infty, L_{\|} / L_{\perp}$ fixed.

Finite-size effects are different in these two cases. The latter limit leads to the infinite volume values of thermodynamic quantities in the three-dimensional three-state Potts model, while the former gives the limiting results for an infinitely long tube and the limit $L_{\perp} \rightarrow \infty$ has still to be taken in order to reach the thermodynamic limit. In particular, quantities such as the energy density or magnetization will be analytic functions of $\beta$ when only the first limit is taken, while they develop a discontinuity in the second case. These qualitative differences 
are obvious from the results obtained for the internal energy,

$$
E=\frac{1}{3 L_{\perp}^{2} L_{\|}}\left\langle\sum_{x, e} \delta_{s_{x}, s_{x+e}}\right\rangle
$$

shown in fig. 1. In particular the results on lattices with par.b.c. (fig. 1a and b) exemplify the different limits discussed above. We also note that the different boundary conditions lead to consistent results for small $\beta$, but show a strong dependence on the boundary conditions in the ordered phase, i.e. at large $\beta$. This is as expected; in the disordered phase, the memory of the boundary conditions does get destroyed through a disordered phase that appears in an intermediate effective volume,

$$
V_{\mathrm{eff}}\left(k_{\mathrm{c}}\right)=L_{\perp}^{2}\left(L_{\|}-2 k_{\mathrm{c}}\right)
$$

with coordinates $x_{\|}$restricted to the region $k_{\mathrm{c}} \leqslant x_{\|} \leqslant L_{\|}-k_{\mathrm{c}}$. Parallel and rotated boundary conditions thus become equivalent in the disordered phase. In the ordered phase, however, rot.b.c. enforce the appearance of at least one interface between differently oriented domains, while for par.b.c. (as well as for periodic boundary conditions) a unique ordering of the entire lattice is possible. It is clear that an interface will lead to a smaller internal energy $E$, which is obvious from fig. $1 c$ and $d$. As will be discussed in detail in the next sections, a smaller value of $E$ corresponds to a larger free energy, which in turn reflects the presence of interfaces.

The similarity between par.b.c. and periodic boundary conditions is also obvious from the comparison of our data with results from simulations on cubic lattices [16]. In order to compare the thermodynamics on lattices with fixed boundaries and cylindrical geometry with that of cubic lattices with periodic boundary conditions, the energy density $E$ must be analyzed in the smaller, effective volume defined in eq. (7). As the ferromagnetic influence of the boundaries is not expected to be significant at distances larger than the correlation length, we have chosen $k_{\mathrm{c}}$ to be equal to the maximal correlation length measured in the critical region [16], i.e. $k_{\mathrm{c}} \simeq \xi_{\mathrm{c}} \simeq 10$. In fig. 2 we compare results for the internal energy $E$ from simulations on lattices of size $24^{2} \times 96$ using both sets of fixed boundary conditions with those from simulations on $24^{3}$ and $48^{3}$ lattices using periodic boundary conditions [16]. This shows that results from simulations with rot.b.c. deviate strongly from the large-volume results $\left(48^{3}\right)$, even after suppressing the strongly distorted regions close to the boundaries.

The interpolating curves shown in figs. 1 and 2 are obtained using the multiplehistogram technique of Ferrenberg and Swendsen (FS-interpolation) [23]. They are based on high-statistics measurements at several $\beta$ values in the interval $\beta \in$ $(0.548,0.552)$. We use this approach to extract also other observables. In particular, 

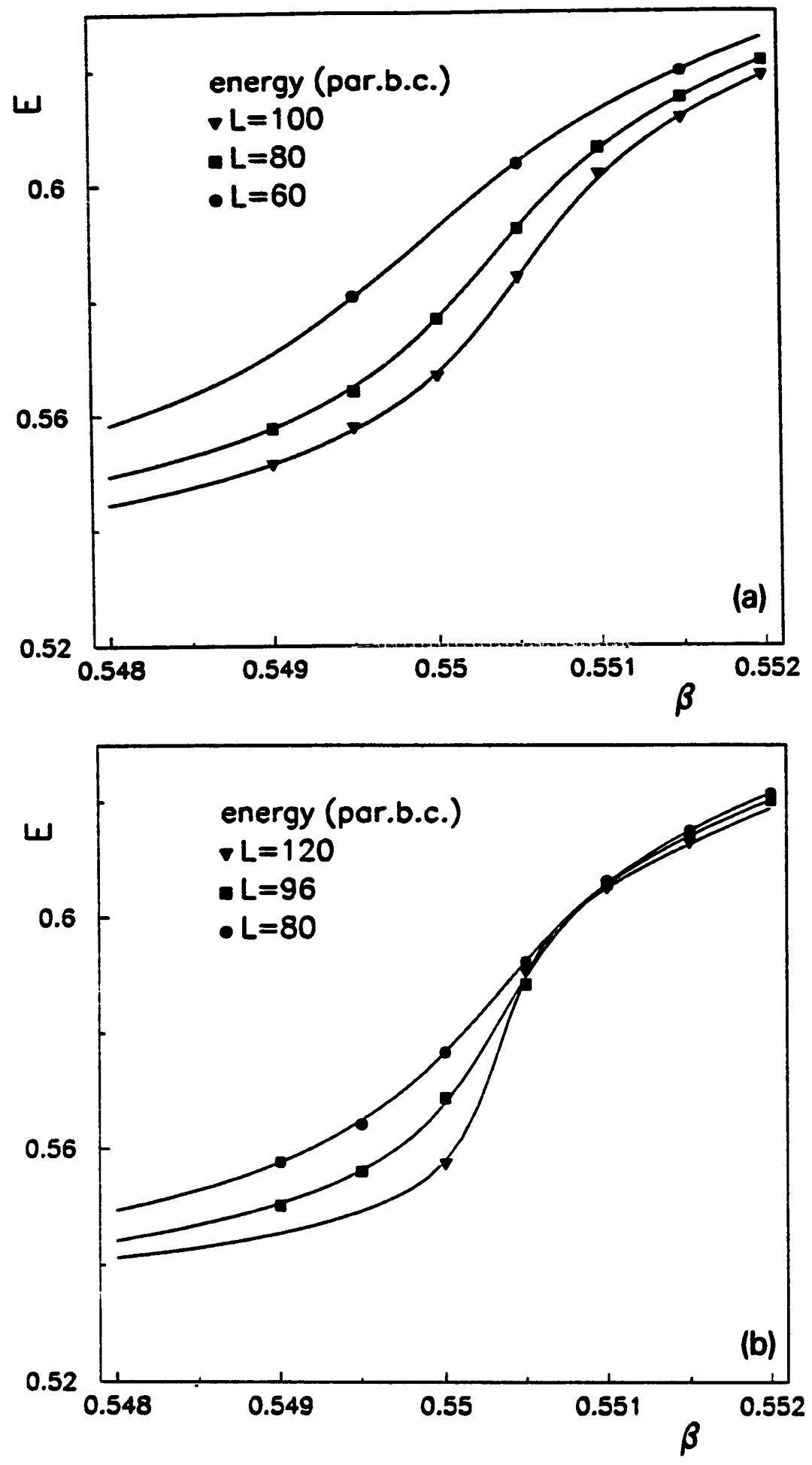

Fig. 1. The internal energy $E$ on lattices of size (a) $20^{2} \times L_{\|}$with $L_{\|}=60,80$, and 100, (b) $L_{\|} / L_{\perp}=4, L_{\perp}=20,24,30$ with par.b.c. and the same for rot.b.c. (c) and (d), respectively. Averages are formed with $k_{\mathrm{c}}=1$. The solid curves are interpolations based on the method described in ref. [23]. 

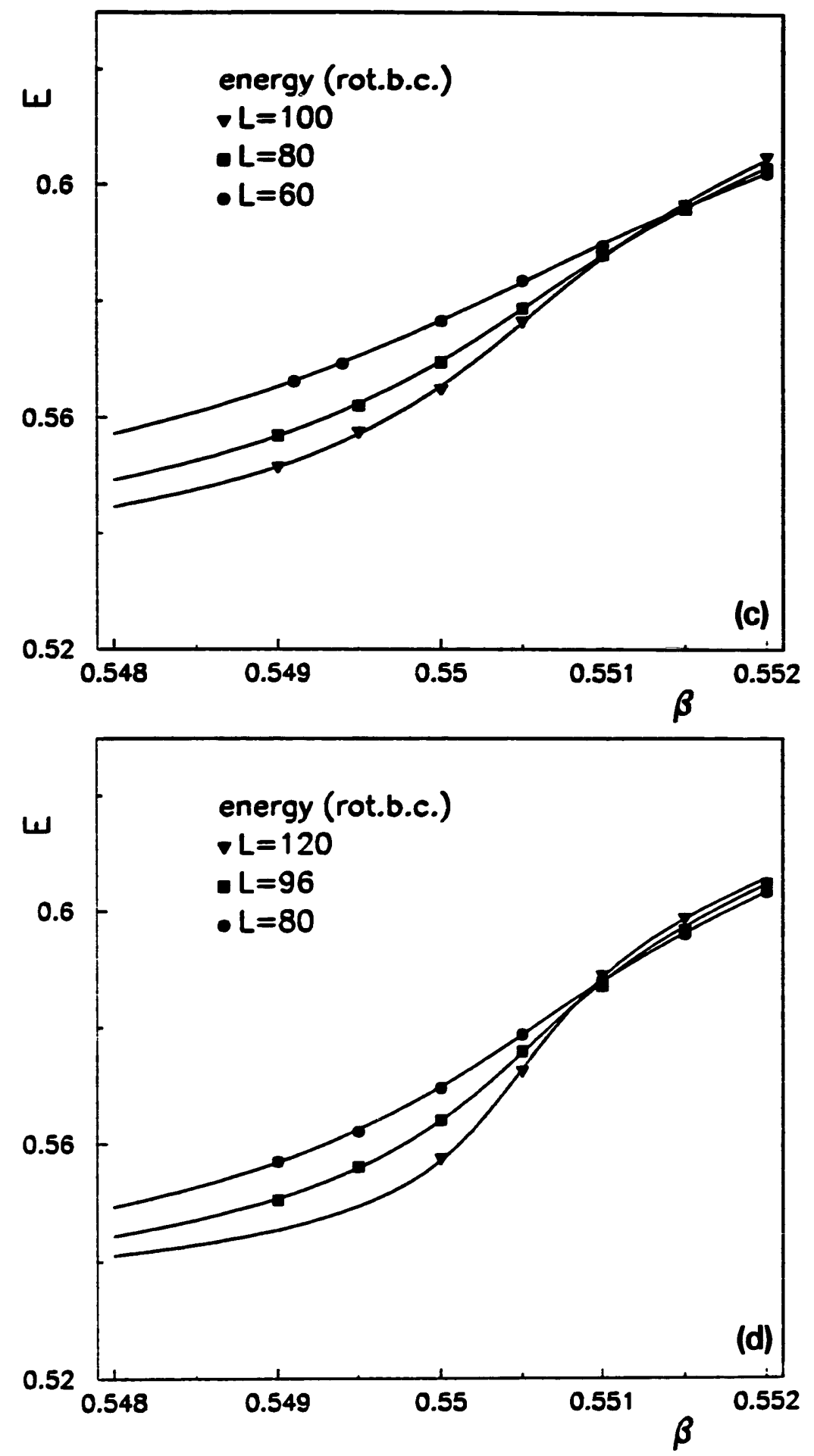

Fig. 1. (continued). 


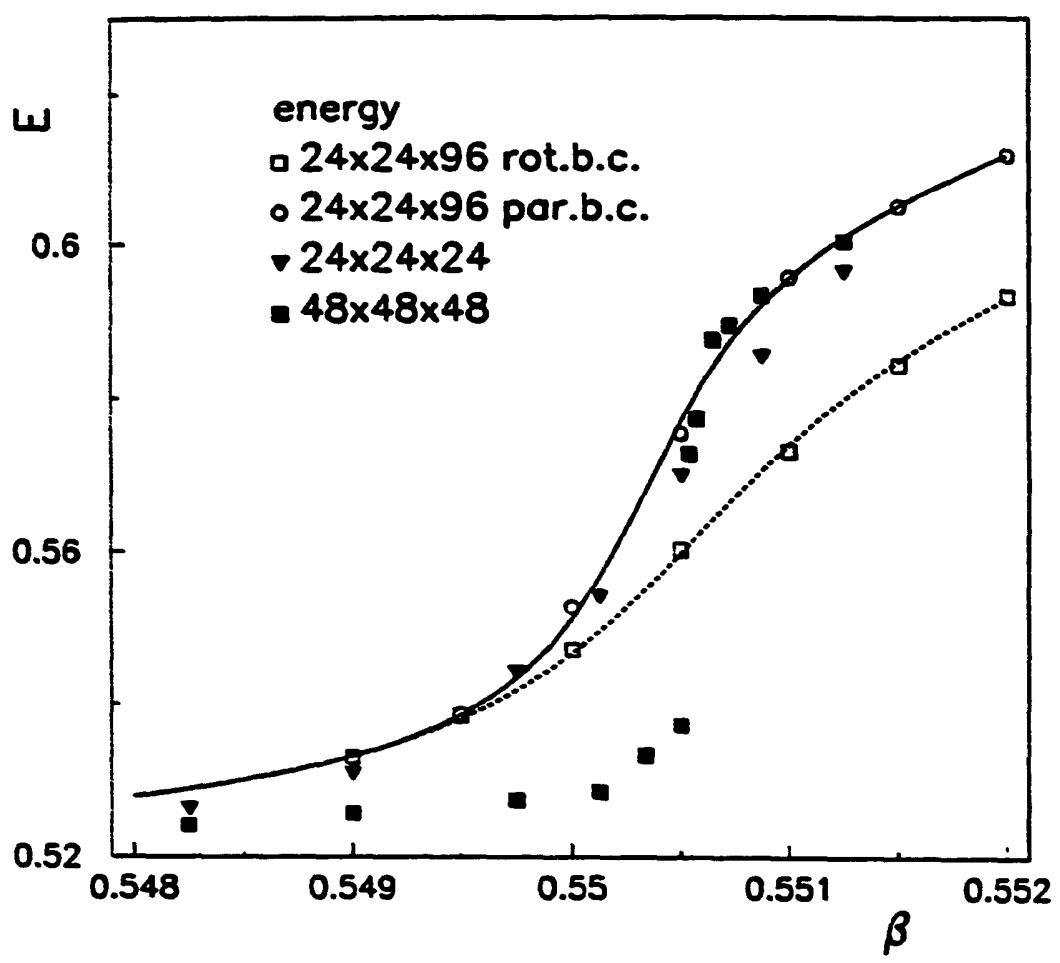

Fig. 2. The internal energy $E$ on lattices of size $24^{2} \times 96$ for rot.b.c. (open squares) and par.b.c. (open circle). Averages are formed in an effective volume $V_{\text {eff }}(10)$. Also shown are results from $L^{3}$ lattices with periodic boundary conditions for $L=24$ (full triangles) and 48 (full squares).

we will use it to extract the free energy of the system. This will be discussed in detail in sect. 5. Here we use the multiple-histogram technique to extract the critical couplings on finite lattices from peaks in response functions such as the specific heat

$$
C_{\mathrm{V}}=\frac{1}{L_{\perp}^{2} L_{\|}}\left(\left\langle H^{2}\right\rangle-\langle H\rangle^{2}\right)
$$

and cumulants of the hamiltonian [24]

$$
V_{L}=1-\frac{\left\langle H^{4}\right\rangle}{3\left\langle H^{2}\right\rangle^{2}}
$$

A behavior similar to that for the internal energy and related quantities is found for the magnetization, which we define in a somewhat unconventional way, as we are particularly interested in the spatial dependence of the magnetization. We first define, for each plane orthogonal to the long axis of the cylinder, the magnetization per plane,

$$
m\left(x_{\|}\right)=\frac{1}{L_{\perp}^{2}}\left(\frac{3}{2} \max \left(n_{0}, n_{1}, n_{2}\right)-\frac{1}{2}\right)
$$




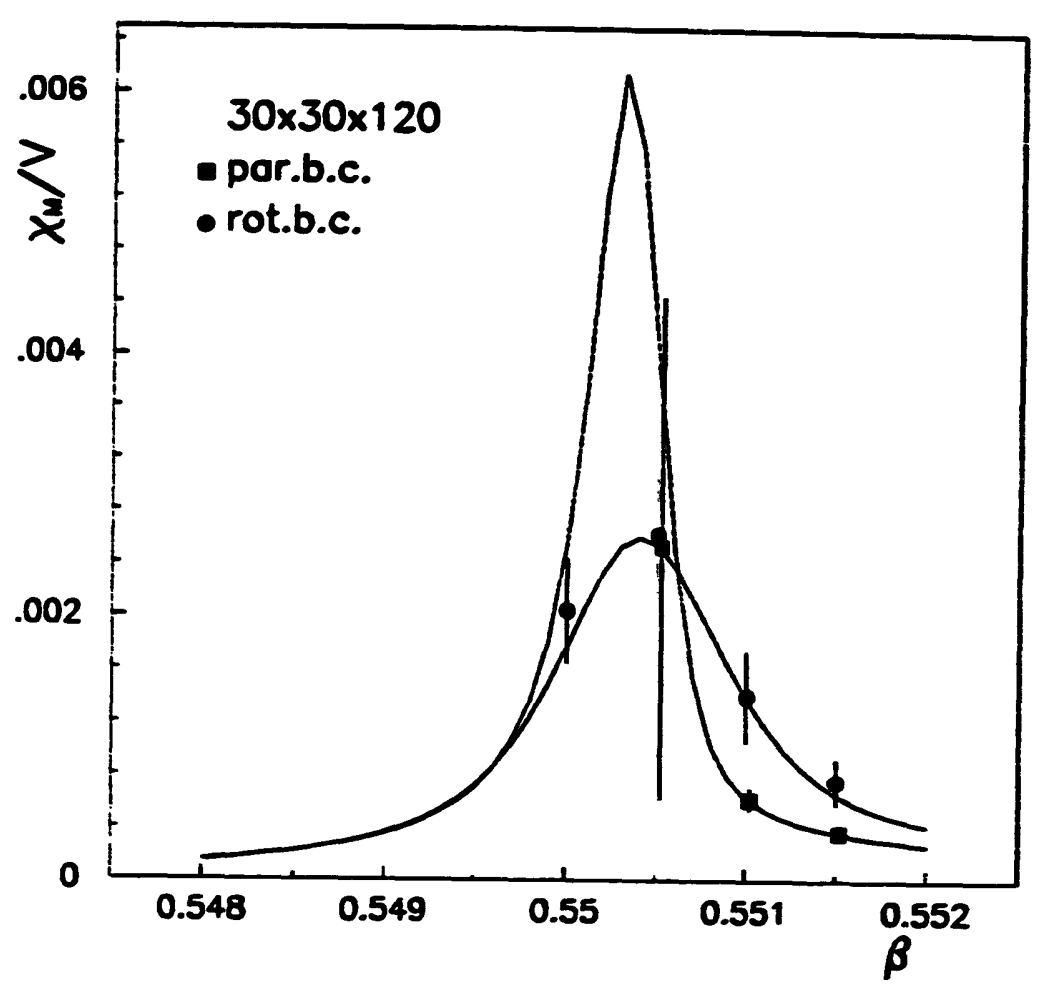

Fig. 3. The magnetic susceptibility on the largest lattice studied $\left(30^{2} \times 120\right)$ for par.b.c. (upper curve) and rot.b.c. (lower curve). The curves are Ferrenberg-Swendsen interpolations based on measurements in the interval $\beta \in(0.550,0.5515)$.

Here $n_{i}$ is the number of occurrences of the state $i$ on sites in a given transverse plane at $x_{\|}$. Next, the average over the length of the cylinder gives the volume average of the magnetization:

$$
M=\frac{1}{L_{\|}-2} \sum_{x_{\|}=2}^{L_{\|}-1} m\left(x_{\|}\right) .
$$

From this we also extract the magnetic susceptibility

$$
\chi_{\mathrm{M}}=L_{\perp}^{2} L_{\|}\left(\left\langle M^{2}\right\rangle-\langle M\rangle^{2}\right)
$$

Some results for $\chi_{M}$ are shown in fig. 3. Also here the smaller peak value, obtained for the case of rot.b.c., reflects the fact that these boundary conditions lead to results that deviate strongly from the infinite-volume results. The critical couplings, however, are close to the infinite-volume result given in eq. (3). From the location of the peaks and their width we find $\beta_{c}=0.5503(2)$ for par.b.c. and $0.5504(5)$ for rot.b.c. The critical couplings extracted from the peaks in $C_{V}$ and $V_{L}$ are in agreement with the above estimates. In fact, on all our lattices with $L_{\perp} \geqslant 20$ and $L_{\|} \geqslant 80$ we find critical couplings in the range $\beta_{c}=0.5502-0.5506$. 
We would like to stress at this point that the different finite-size effects seen in the behavior of $E$ in the case of par.b.c. for the two different limits discussed above (figs. la and 1b) have important consequences for the behavior of the surface tension. The internal energy $E$ decreases monotonically with increasing $L_{\|}$ for fixed $L_{\perp}$, which will lead to a decreasing estimate for the surface tension in this limit. However, if we consider the infinite volume limit for fixed $L_{\|} / L_{\perp}$ we find that $E$ rises (decreases) above (below) $\beta_{c}$ for increasing $L_{\|}$, i.e. $E$ starts developing a discontinuity at $\beta_{\mathrm{c}}$. As we will extract the surface tension from an integral over the internal energy (see sect. 4), we will obtain lower bounds for it in the ordered phase, when taking the infinite-volume limit in the latter way. For this reason we will concentrate in the following on this limiting procedure.

\section{Order-order interface statistics}

In the ordered phase the dominant contribution to the free energy arises from configurations with a unique direction of magnetization, unless different domains are forced to appear by a conflicting choice of boundary conditions. For instance, the rotated boundary conditions, defined in eq. (5), demand the appearance of at least one interface between differently magnetized domains. The appearance of several interfaces is thermodynamically suppressed, since the additional surface energy increases the free energy of the system. For the parallel boundary conditions, defined in eq. (4), the first configuration with differently oriented domains requires already two interfaces. Note that this is also the case for a system with periodic boundary conditions. This explains also why the finite-size effects for systems with periodic and parallel (fixed) boundary conditions are similar.

In our simulations, with fixed boundary conditions, interfaces can appear on any transverse plane of the lattice, and the frequency of their occurrence reflects the relative energies of such configurations. In order to get some idea about the structure of the interfaces and their relative rates, we analyze in more detail the magnetization of transverse planes. The magnitude of this magnetization per plane is given by eq. (10). Its orientation can again be characterized by a Potts variable $S_{x_{\|}}$taking on integer values $i=0,1$ or 2 :

$$
S_{x_{\|}}=\left\{i: \max \left(n_{0}, n_{1}, n_{2}\right) \text { in a transverse plane at } x_{\|}\right\},
$$

where $n_{i}$ is the number of occurrences of the state $i$ in that plane. When $S_{y} \neq S_{z}$ on neighboring planes $y$ and $z$, we will count this as a bare domain wall located between these two planes. Of course, there is a certain ambiguity in assigning a discrete spin variable to a transverse plane. When the number of spins in two directions is nearly equal, this may lead to the assignment of spurious interfaces, which we will call defects in the following. In general, each defect introduces two 


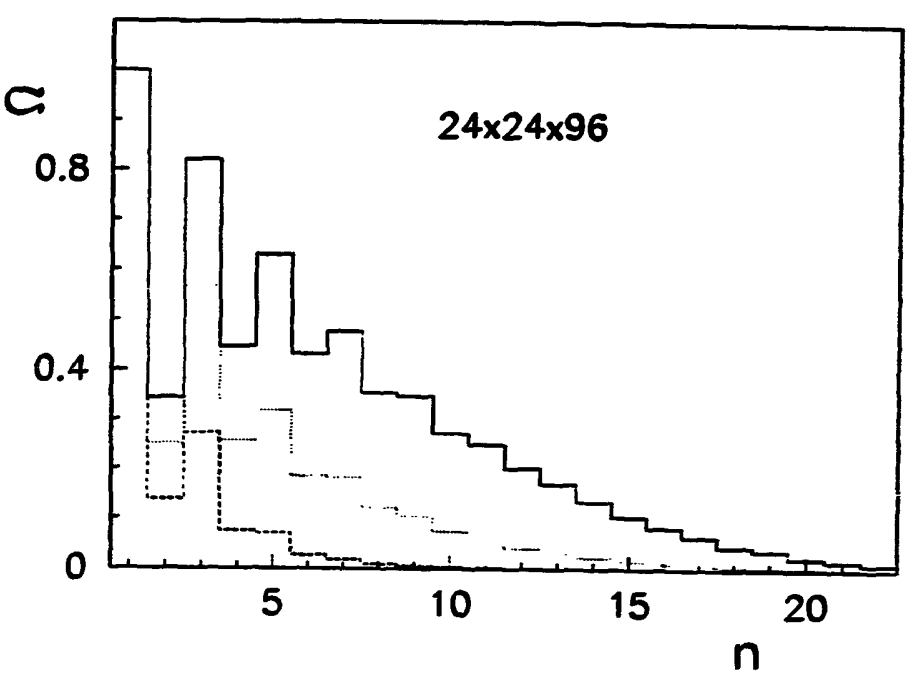

Fig. 4. The relative frequency $\Omega \equiv \Omega_{n} / \Omega_{1}$ of occurrence of the configurations with $n$ bare domain walls on a lattice of size $24^{2} \times 96$ with rot.b.c. The histograms are for $\beta=0.5505$ (solid line), $\beta=0.551$ (dotted line), and $\beta=0.552$ (dashed line); the normalization conştants are $\Omega_{1}=0.2543,0.4370$ and 0.7309 , respectively.

bare domain walls into the configuration. We expect that for $\beta \gg \beta_{c}$ the number of defects will be small and the interpolation between the two boundary values will proceed through the minimal possible number of domain walls.

Already at $\beta=0.552$ we found that defects are indeed strongly suppressed. In our simulations with rot.b.c. on a $24^{2} \times 96$ lattice, for instance, $62 \%$ of the configurations are bare one-wall configurations, while in the case of par.b.c. up to 99.4\% of the configurations have no domain walls at all in the above sense. This large fraction of ground-state configurations in the case of par.b.c. is qualitatively understood as two additional interfaces have to be present in order to obtain a non-trivial domain structure.

In fig. 4 we show, on a lattice of size $24^{2} \times 96$ with rotated boundary conditions, the distribution of bare domain walls, defined in terms of the variables in eq. (13), at $\beta=0.5505,0.551$, and 0.552 . It is obvious from this figure that with increasing coupling, $\beta>\beta_{c}$, configurations with several bare domain walls are more and more suppressed. It is interesting to note that even in the critical region the one-wall configurations dominate on lattices of this size.

We further note that the even and odd wall sectors seem to follow a different pattern. Configurations with an even number of walls are suppressed on lattices with rot.b.c. In fact, the appearance of the even wall sector is peculiar to a $Z(N)$ theory with $N \geqslant 3$. For a $\mathrm{Z}(2)$ symmetric theory it would be completely missing. It also is intuitively clear that defects, which predominantly appear close to a genuine interface, are preferably magnetized in one of the directions selected by the boundary conditions. An even wall configuration can only occur if three neighboring planes have their net magnetization pointing to the three different $Z(3)$ sectors 
TABLE 1

Predictions of the dilute defect model for the frequency of bare domain walls in the odd wall sector. The probabilities $p_{1}$ and $p_{\mathrm{d}}$, entering eq. (14), have been fixed using the Monte Carlo data for $N=0$, i.e. $P_{1}^{\text {exp }}$

\begin{tabular}{ccccc}
\hline & \multicolumn{2}{c}{$\beta=0.551$} & \multicolumn{2}{c}{$\beta=0.552$} \\
\cline { 2 - 5 }$N$ & $\boldsymbol{P}_{2 N+1}^{\text {cxp }}$ & $P_{2 N+1}^{\text {model }}$ & $P_{2 N+1}^{\text {exp }}$ & $P_{2 N+1}^{\text {model }}$ \\
\hline 0 & 0.4370 & 0.4370 & 0.7309 & 0.7309 \\
1 & 0.2480 & 0.2460 & 0.1993 & 0.1967 \\
2 & 0.1392 & 0.1385 & 0.0519 & 0.0529 \\
3 & 0.0800 & 0.0780 & 0.0132 & 0.0142 \\
4 & 0.0453 & 0.0439 & 0.0034 & 0.0038 \\
5 & 0.0244 & 0.0247 & 0.0010 & 0.0010 \\
6 & 0.0135 & 0.0139 & 0.0003 & 0.0003 \\
7 & 0.0072 & 0.0078 & - & - \\
8 & 0.0030 & 0.0044 & - & - \\
9 & 0.0016 & 0.0025 & - & - \\
\hline
\end{tabular}

of the Potts model. The larger number of odd domain walls is thus qualitatively understood.

We also find that their relative rates are well described by a dilute defect approximation, i.e. the simplest ansatz is to assume that one has only one genuine interface and a few dilute defects, which occur with probability $p_{\mathrm{d}}$ in the vicinity of this interface. Within the odd sector the probability for having $2 N+1$ bare domain walls (one genuine interface and $N$ defects) is then given by

$$
P_{2 N+1}=p_{1} p_{\mathrm{d}}^{N}, \quad N \geqslant 0 .
$$

The probability $p_{1}$ for having a single interface without additional defects can be taken from our data. The completeness relation $p_{1}+p_{\mathrm{d}}=1$ then gives a prediction for the defect probability. In the range $0.5505 \leqslant \beta \leqslant 0.552$ we find that $p_{\mathrm{d}}$ decreases from 0.75 to 0.27 . At $\beta=0.551$, for instance, we obtain $p_{1}=0.437$ from our data, which leads to $p_{\mathrm{d}}=0.563$ for the defect probability. In table 1 we compare the measured probabilities for the occurrence of $(2 N+1)$-wall configurations with predictions of the dilute defect model characterized by eq. (14). The agreement is surprisingly good and it remains still acceptable on the top of the transition region at $\beta=0.5505$.

Similarly we can describe the even wall sector in the case of parallel boundary conditions. It is, however, less straightforward to characterize the statistics in the remaining cases, i.e. the even (odd) wall sector in the case of rot.b.c. (par.b.c.). Here one has to give up the assumption of dilute defects. As described above one has to take into account that the fundamental object is characterized by the magnetization on three neighbouring planes. In any case the contribution of these 
sectors is suppressed statistically and of little importance for the quantitative analysis of the surface tension. We thus will refrain from a more detailed analysis at this point.

The success of our dilute defect model shows that most of the multiple wall configurations are in fact modifications of the dominant domain structure imposed by the boundary conditions, i.e. one-wall (no-wall) configurations in the case of rot.b.c. (par.b.c.). As a consistency check we also extended the above model by allowing for an intrinsic probability for three genuine interfaces. As expected, rather small probabilities were found for such configurations. We thus conclude that in our above example $\left(V=24^{2} \times 96, \beta=0.551\right)$ the whole odd sector represents a single interface and its weight is actually $84 \%$ in the total sample.

Since interfaces can appear in transverse planes located at arbitrary $x_{\|}$, their presence is not directly evident from an analysis of the average magnetization per plane. However, we can easily see the dominance of single-wall configurations, by dividing the whole data sample into subclasses, $\mathrm{C}\left(x_{\min }\right)$, consisting of all configurations for which the plane with minimal magnetization is located at a fixed position, $x_{\|}=x_{\min }$. The resulting magnetization profile for $x_{\min }=L_{\|} / 2$ is shown in fig. 5 .

A comparison between the profiles obtained from the total data sample (fig. 5a) with that of the subset of configurations with only a single domain wall (fig. 5b) shows that the additional defects contributing to the former case just lead to a widening of the kink structure. The difference of the two profiles at $\beta=0.5505$ is quite instructive: the subset of one-wall configurations, which represents about $50 \%$ of all configurations having their minimum at the prescribed point, is still building up an order-order interface. These are the multiple wall configurations which drive the system at this coupling into the disordered phase. The dominance of a single domain wall already for $\beta \geqslant 0.551$ is, however, obvious from these figures. Note that the profiles found are very similar to those obtained in a mean field calculation [13].

\section{Surface tension and action asymmetry}

The discussion in sect. 3 has shown that in the symmetry-broken phase an overwhelming number of configurations has the domain wall structure of the ground state imposed through the choice of boundary conditions, i.e. in the case of rot.b.c. we find dominantly a single interface whereas in the case of par.b.c. there appears no interface at all. We should thus be able to determine the surface tension at a given coupling in the symmetry broken phase by taking the difference between the free energies of the two systems with different fixed boundary conditions. The free energy is related to the internal energy $\langle H\rangle$ by the well-known relation

$$
\beta, \mathscr{F}(\beta)-\beta_{0}, \mathscr{F}\left(\beta_{0}\right)=\int_{\beta_{01}}^{\beta}\left\langle H\left(\beta^{\prime}\right)\right\rangle \mathrm{d} \beta^{\prime},
$$



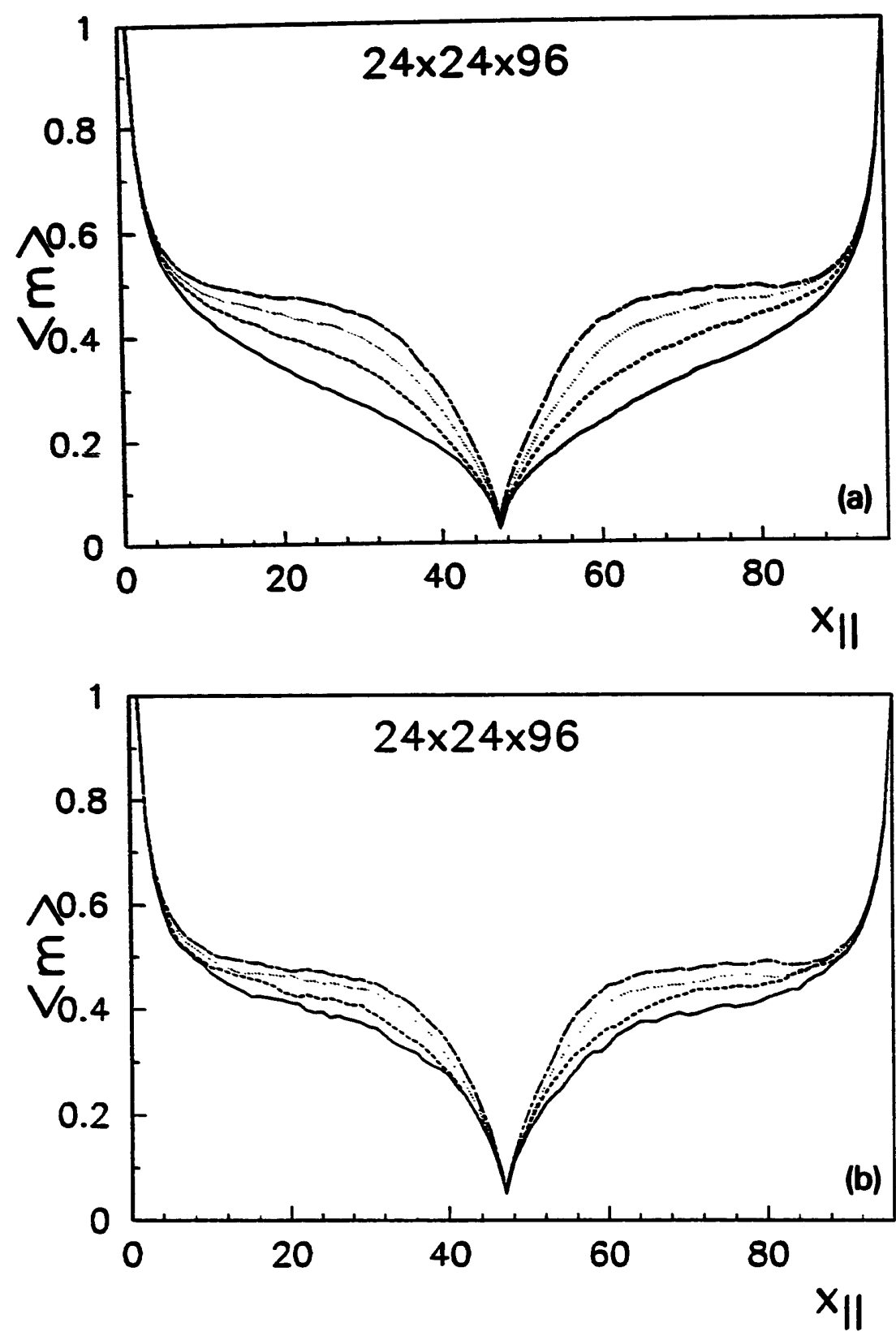

Fig. 5. The magnetization per plane, $\left\langle m\left(x_{\|}\right)\right\rangle$, on a $24^{2} \times 96$ lattice with rot.b.c. at various values of $\beta$ averaged over configurations with the minimum of $\left\langle m\left(x_{\|}\right)\right\rangle$fixed at $x_{\|}=48$. Shown are spin profiles for $\beta=0.552$ (dash-dotted), $\beta=0.5515$ (dotted), $\beta=0.551$ (dashed) and $\beta=0.5505$ (solid). The curves are drawn by piecewise linear interpolation between the central values of $\left\langle m\left(x_{\|}\right)\right\rangle$on neighbouring planes. Typical errors are of the order of $10 \%$ of the central values in the region of the tip of the kink and $1 \%$ away from it. Shown are averages over a subset selected from the full sample (a), and from the configurations having only a single bare domain wall (b). 
where

$$
\beta \cdot F(\beta)=-\ln \sum_{\left\{s_{x}\right\}} \exp (-\beta H)
$$

In the symmetric phase the free energy is independent of the kind of fixed boundary 'conditions chosen; at a certain distance from the boundary planes an inner disordered phase will build up and in this way the information about the type of fixed boundary conditions chosen on one side of the lattice is lost. This is also obvious from fig. 2 , which shows that $\langle H\rangle$ is independent of the boundary conditions for $\boldsymbol{\beta}<\boldsymbol{\beta}_{\mathrm{c}}$. One can thus choose a coupling $\boldsymbol{\beta}_{\mathbf{0}}$ in the disordered phase where none of the boundary conditions is able to maintain an interface $\left[\bar{\nexists}_{\text {rot }}\left(\beta_{0}\right)\right.$ $=\mathscr{F}_{\text {par }}\left(\beta_{0}\right)$ ]. One then finds for the interface tension in the symmetry broken phase

$$
\alpha_{\text {ord }}=\frac{1}{L_{\perp}^{2}}\left(\mathscr{\not}_{\text {rot }}(\beta)-\mathscr{F}_{\text {par }}(\beta)\right)
$$

The surface tension appears also in the second law of thermodynamics as a response to infinitesimal variations in the cross section, $A$, of the interface

$$
\mathrm{d} . \tilde{F}=-p \mathrm{~d} V+\alpha \mathrm{d} A+S \mathrm{~d} T \text {. }
$$

The scalar product $\alpha \mathrm{d} \boldsymbol{A}$ reflects the effect of cross-section variations for all three independent orientations.

The boundary conditions in our case trigger an interface orthogonal to the I-direction. The axial symmetry of the system makes it natural to consider scale changes of the form

$$
x_{\perp, i} \rightarrow x_{\perp, i}\left(1+\delta \lambda_{\perp}\right), \quad x_{\|} \rightarrow x_{\|}\left(1+\delta \lambda_{\|}\right),
$$

which lead to

$$
\delta A=2 \delta \lambda_{\perp} A, \quad \delta V=\left(2 \delta \lambda_{\perp}+\delta \lambda_{\|}\right) V
$$

Let us fix the temperature and the ratio

$$
\frac{\delta \lambda_{\|}}{\delta \lambda_{\perp}}=z
$$

Then one finds from eq. (18)

$$
\frac{\mathrm{d} . \widetilde{\mathcal{F}}}{\mathrm{d} \lambda_{\perp}}=-p V(z+2)-2 \alpha A .
$$


With the application of two independent scale transformations the volume dependence can be eliminated. For definiteness we consider the cases $z=0$ and 1 , respectively:

$$
\left.\frac{3}{2} \frac{\mathrm{d} \mathscr{F}}{\mathrm{d} \lambda_{\perp}}\right|_{\Sigma=0}-\left.\frac{\mathrm{d} \mathscr{F}}{\mathrm{d} \lambda_{\perp}}\right|_{\Sigma=1}=\alpha A
$$

Using the definition of the free energy, eq. (16), one can express the left-hand side of eq. (23) as

$$
\left.\frac{3}{2}\left\langle\frac{\mathrm{d} H}{\mathrm{~d} \lambda_{\perp}}\right\rangle\right|_{z=0}-\left.\left\langle\frac{\mathrm{d} H}{\mathrm{~d} \lambda_{\perp}}\right\rangle\right|_{\Sigma=1}=\alpha A .
$$

Near the transition point $\boldsymbol{\beta}_{\mathrm{c}}$ one can use ideas related to the renormalization group (RG) for finding the derivatives on the left-hand side. A first-order transition with coexistence of phases has the feature that in each distinct phase the linearized RG transformation has a distinct eigenvector with the eigenvalue $\lambda^{d}$ (where $\lambda$ is the scale change and $d$ the dimensionality of the system). Discontinuities arise by the passage of the state of the system from one of the eigenvectors to the other degenerate with it [25]. In a transition with latent heat, the hamiltonian has non-zero projection on these eigenvectors. At least for isotropic rescaling of the system one therefore expects near the transition point

$$
\left.\left\langle\frac{\mathrm{d} H}{\mathrm{~d} \lambda}\right\rangle\right|_{T \approx T_{\mathrm{c}}}=- \text { const. } \times\langle H\rangle .
$$

For the anisotropic case with $z=0$ a natural assumption is that only those pieces of the hamiltonian will transform, which contain couplings parallel to the rescaled directions:

$$
\left.\left\langle\frac{\mathrm{d} H_{\perp}}{\mathrm{d} \lambda_{\perp}}\right\rangle\right|_{T=T_{\mathrm{c}}}=- \text { const. } \times\left\langle H_{\perp}\right\rangle,\left.\quad\left\langle\frac{\mathrm{d} H_{\|}}{\mathrm{d} \lambda_{\perp}}\right\rangle\right|_{T \approx T_{\mathrm{c}}}=0 .
$$

Using eqs. (25) and (26) one arrives at a relationship conjectured by Kajantie et al. $[6,19]$

$$
\alpha=\text { const. } \times . \propto /,
$$

where the action asymmetry $\mathscr{G}$ is defined by

$$
\begin{aligned}
\mathscr{W} & =\frac{1}{L_{\perp}^{2}}\left(\left\langle H_{\|}\right\rangle-\frac{1}{2}\left\langle H_{\perp}\right\rangle\right) \\
& =\frac{1}{2 L_{\perp}^{2}} \sum_{x}\left\langle\delta_{s_{x}, s_{x+e_{1}}}+\delta_{s_{x}, s_{x+e_{2}}}-\delta_{s_{x}, s_{x+e_{3}}}-\delta_{s_{x}, s_{x-e_{3}}}\right\rangle .
\end{aligned}
$$


Rummukainen [19] proposed for the proportionality constant appearing in eq. (27) a value of about 1.4. The scaling argument given above, on the other hand, would suggest unity.

Away from the transition region we have no guidance to relate $\&$ to $\alpha$. RG transformations are not universal far away from the fixed point, and different transformations would thus lead to different trajectories in the coupling space. Therefore it is better to consider $\mathscr{H}$ and $\alpha$ as two independent global characteristics of the interface. In case of the existence of an underlying continuum theory, the scaling towards the continuum will relate the two quantities as discussed in refs. $[6,10]$.

The independence of $\mathscr{O}$ and $\alpha$ is also clear from their low-temperature $(\beta \gg 1)$ series expansions which to lowest order look like

$$
\begin{aligned}
& \alpha_{\text {ord }}=1-\frac{2}{\beta} \exp (-4 \beta)+O(\exp (-6 \beta)), \\
& \mathscr{H}_{\text {ord }}=1-4 \exp (-4 \beta)+O(\exp (-6 \beta))
\end{aligned}
$$

The action asymmetry for the order-disorder transition has been studied in ref. [19]. Using our conventions for the normalization of $\mathscr{H}$, the result of this analysis was

$$
\mathscr{Q}_{\mathrm{dis}}=(4 \pm 2) \times 10^{-3}
$$

An advantage of $\mathscr{H}$ is that it can, in principle, be measured using exclusively the rotated boundary conditions, while a determination of $\alpha$ from the free energies requires the use of two samples. However, it turns out to be very useful to extract $\mathscr{H}$ also from differences of simulations between rot.b.c. and par.b.c., as this allows finite-size effects to be drastically reduced.

\section{Numerical results for surface tension and action asymmetry}

We start the presentation of our numerical results with the surface tension calculated from eq. (17). The free energies on lattices with different fixed boundary conditions are calculated from eq. (15), using for the starting point of the integration a value $\beta_{0}$ in the symmetric phase; in other words we normalize the free energy to be zero at $\beta_{0}=0.549$. In fig. 6 we show the results of an integration of our data for the energy density $E$ (fig. 1), using a Simpson formula. The data points correspond to an integration of the energies measured at $\beta$-values separated by $\Delta \beta=0.0005$. Also shown in this figure are the results of a numerical integration of the FS-interpolations for $E$, using a Simpson formula with $\Delta \beta=$ 0.0001 . For each lattice we show on the last data point the cumulative error coming from a summation of the individual errors on $E$. These errors are of the 


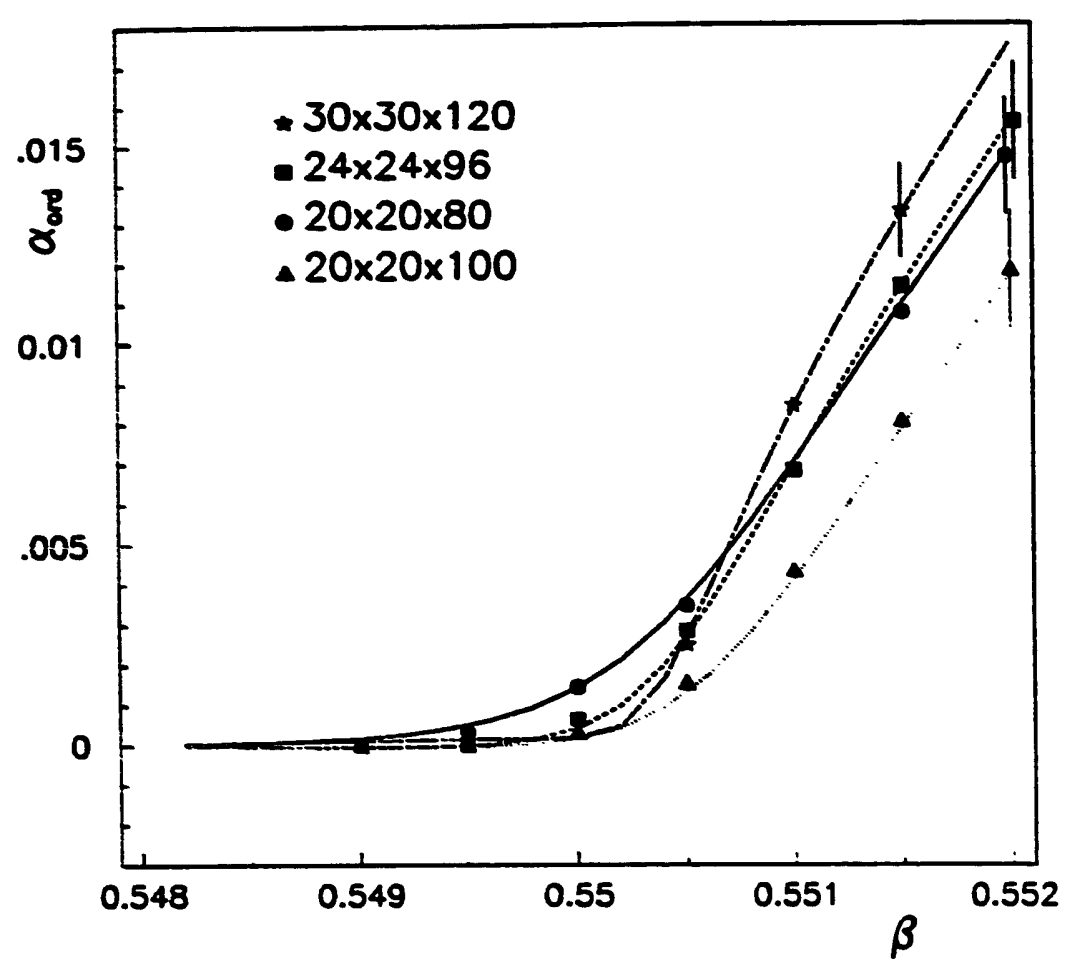

Fig. 6. Surface tension from the difference of free energies on various lattices versus $\beta$. Results for $30^{2} \times 120$ (dash-dotted line), $24^{2} \times 96$ (dashed line), $20^{2} \times 80$ (solid line), and $20^{2} \times 100$ (dotted line) are presented. The data points and the curves have been obtained from a Simpson integration of the internal energy. Details of the integration procedure as well as the error bars are given in the text.

order of $10 \%$. However, they clearly overestimate the true error, since they assume a strong correlation between individual measurements of $E$. The very good agreement of the estimates obtained in both ways reflects the mutual consistency of our data.

We note that the curves corresponding to the set of lattice sizes with $L_{\|}=4 L_{\perp}$ cross in the vicinity of the infinite volume critical coupling, leading to a larger surface tension on larger lattices in the ordered phase. The crossing point approaches $\beta_{c}$ from above with increasing lattice size. This behavior is similar to that of the correlation length close to $\beta_{c}[16]$ and supports the expectation that in the limit $L_{\perp} \rightarrow \infty$ a discontinuity will build up at $\boldsymbol{\beta}_{\mathrm{c}}$. It further suggests that our results for $\alpha_{\text {ord }}\left(\beta_{\mathrm{c}}\right)$, obtained on our smallest lattice $\left(20^{2} \times 80\right)$, give a lower bound for the surface tension at $\boldsymbol{\beta}_{\mathrm{c}}$. We find

$$
\alpha_{\text {ord }}\left(\beta_{\mathrm{c}}\right) \geqslant 0.0048 \pm 0.0003 .
$$

The relatively large errors on $\alpha_{\text {ord }}$ for $\beta>\beta_{\mathrm{c}}$, however, do not allow a detailed finite-size analysis for $\alpha_{\text {ord }}$ above $\beta_{\mathrm{c}}$. We thus cannot extrapolate to the infinitevolume limit above $\beta_{\mathrm{c}}$, which would allow us to give also an upper bound on $\alpha_{\text {ord }}$. It also seems that finite-volume effects depend strongly on the ratio $L_{\|} / L_{\perp}$. This 
is obvious from the values of the surface tension obtained from our simulation on a $20^{2} \times 100$ lattice, i.e. for $L_{\|}=5 L_{\perp}$. The significantly smaller values found for $\alpha_{\text {ord }}$ in this case are, in fact, a direct consequence of the finite-size dependence of $E$ discussed in sect. 3 in connection with fig. 1 . We expect that larger values of $L_{\perp}$ are needed in this case to come close to the infinite volume limit.

An other interesting feature of the data shown in fig. 6 is the nearly linear rise of the surface tension in the ordered phase for $\beta \geqslant 0.551$. This agrees with the behavior found from the mean-field solution [13]. As the data from all our lattices with $L_{\|}=4 L_{\perp}$ agree within statistical errors for $\beta>0.551$ we may use this data sample to get a straight line fit for $\alpha_{\text {ord }}$ in this regime. From a least squares fit we obtain

$$
\alpha_{\text {ord }}(\beta)=0.0040(7)+8.2(1.0)\left(\beta-\beta_{c}\right), \quad \beta \geqslant 0.551
$$

with $\chi^{2} /$ d.o.f. $=0.9$. It is reassuring that the value obtained for $\boldsymbol{\alpha}_{\text {ord }}\left(\beta_{\mathrm{c}}\right)$ from this fit to the whole data sample is in good agreement with the lower bound given in eq. (31). Deviations from this linear behavior occur only on our largest lattice in the critical region, indicating that a discontinuity is building up at $\boldsymbol{\beta}_{\mathrm{c}}$. Of course, we also expect deviations for larger $\beta$, as $\alpha_{\text {ord }}$ has to approach unity asymptotically. It is interesting to note, that the slope found from our data agrees quite well with a mean-field calculation, yielding a value of 8.8 [13].

Let us now turn to the discussion of the action asymmetry defined in eq. (28). The fixed ferromagnetic boundary conditions used by us lead, of course, to a strong distortion of $\%$. These boundary effects can be eliminated (in the largevolume limit) by taking differences of $\mathscr{r}_{\text {rot }}$ and $\mathscr{H}_{\mathrm{par}}$ measured on lattices with rot.b.c. and par.b.c., respectively. We thus obtain the dominant contribution arising from the formation of an interface as

$$
\mathscr{A}_{\mathrm{ord}}=\mathscr{A}_{\mathrm{rot}}\left(k_{\mathrm{c}}=1\right)-\mathscr{A}_{\mathrm{par}}\left(k_{\mathrm{c}}=1\right) .
$$

The results are shown in fig. 7 for our lattices with $L_{\|}=4 L_{\perp}$ both in form of directly subtracted data points and smooth interpolating FS curves. Statistical errors are clearly much larger for $\mathscr{A}_{\text {ord }}$ than for $\alpha_{\text {ord }}$. In fact, on the $30^{2} \times 120$ lattice, measurements were done only in the interval $\beta \in(0.550,0.5515)$ and we observed that the FS-interpolation became unstable outside this interval; it is therefore given only in this region. The data tend to decrease with the volume. However, in view of the larger errors, the significance of this tendency for the larger two volumes is not clear. We thus again use the whole data sample to estimate $\mathscr{A}_{\text {ord }}\left(\beta_{\mathrm{c}}\right)$. Using also here a straight-line fit to the data for $\beta>0.5505$, we obtain

$$
\mathscr{A}_{\text {ord }}\left(\beta_{\mathrm{c}}\right)=0.0080(33)+4.4(3.6)\left(\beta-\beta_{\mathrm{c}}\right), \quad \beta>\beta_{\mathrm{c}}
$$




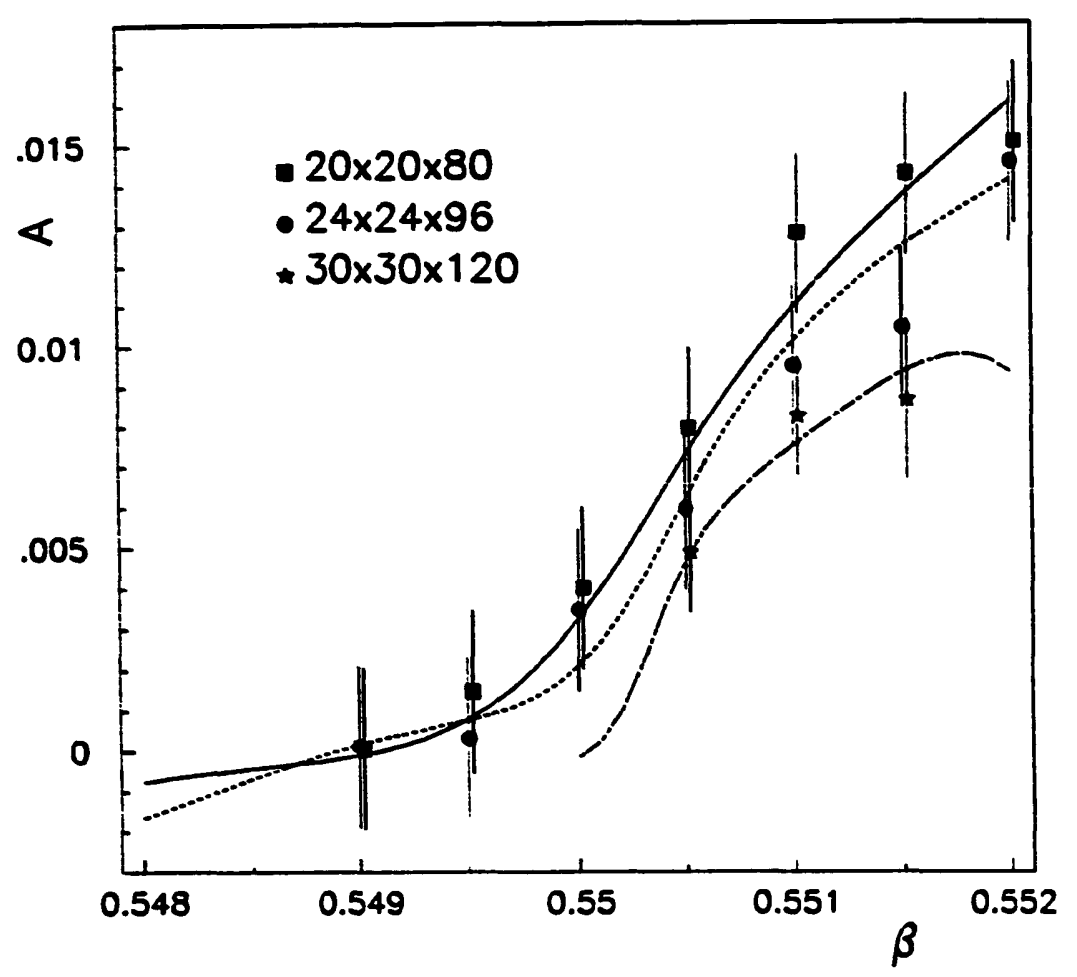

Fig. 7. Action asymmetry $\therefore$ ord as defined in eq. (33). The types of lines are the same as in fig. 6.

A different approach to extract $\mathscr{H}_{\text {ord }}$ makes only use of the measurements performed on lattices with rot.b.c. The action asymmetry is calculated in the effective volume, eq. (7), with $k_{\mathrm{c}}=10$. As discussed above this should eliminate distortions due to the ferromagnetic boundaries. In table 2 the asymmetries obtained with both methods are given, together with the values of the surface tension for all large volumes we have studied.

The agreement between the "subtraction" and the "cut" methods is satisfactory for volumes with fixed $L_{\|} / L_{\perp}(=4)$ ratio. The $20^{2} \times 100$ data obtained in these two ways, however, are substantially different in the low- $\beta$ region.

A direct comparison of $\mathscr{H}_{\text {ord }}$ and $\alpha_{\text {ord }}$ at $\beta=0.552$ (table 2) shows that they agree well within statistical errors. With decreasing $\beta$ the agreement gets worse. This is also reflected by our straight-line fits given in eqs. (32) and (34). However, given the finite-size dependence seen in our data and the large errors in the determination of $\mathscr{Q}_{\text {ord }}$, we cannot rule out that on larger lattices also in this regime $\alpha_{\text {ord }} / \mathscr{A}_{\text {ord }}$ approaches unity.

Let us finally comment about the evidence for complete wetting. If this occurs we would expect that the action asymmetry for order-order interfaces, $\mathscr{A}_{\text {ord }}$, is twice as large as the one for disorder-order interfaces measured in ref. [19]. Comparing our result for $\mathscr{\sigma}_{\text {ord }}\left(\beta_{\mathrm{c}}\right)$ given in eq. (34) with eq. (30) one concludes that the situation is compatible with complete wetting. However, in view of the 
TABLE 2

The surface tension $\alpha_{\text {ord }}$ extracted from differences of the free energy, and the action asymmetry $\because \sigma_{\text {ord }}$ extracted from differences of $\because \sigma_{\mathrm{rot}}$ and $\because \sigma_{\mathrm{par}}\left(k_{\mathrm{c}}=1\right)$ as well as directly from.$r_{\mathrm{rot}}\left(k_{\mathrm{c}}=10\right)$. The stars denote the $\beta$-values at which the free energies have been normalized to zero. The errors on $\because \%$ ord are typically 0.002 for the $k_{\mathrm{c}}=10$ data and twice as large for $k_{\mathrm{c}}=1$, as the latter arise from a difference. The error propagation for $\alpha_{\text {ord }}$ is discussed in the text

\begin{tabular}{|c|c|c|c|c|}
\hline \multirow[b]{2}{*}{$\boldsymbol{\beta}$} & \multirow[b]{2}{*}{$\boldsymbol{L}_{\|}$} & \multirow[b]{2}{*}{$\alpha_{\text {ord }}$} & \multicolumn{2}{|c|}{$\because$ ord } \\
\hline & & & $k_{\mathrm{c}}=1$ & $k_{\mathrm{c}}=10$ \\
\hline \multirow[t]{3}{*}{0.549} & 80 & * & 0.0006 & 0.0056 \\
\hline & 96 & * & 0.0002 & 0.0031 \\
\hline & 100 & * & -0.0004 & 0.0071 \\
\hline \multirow[t]{3}{*}{0.5495} & 80 & 0.0003 & 0.0015 & 0.0054 \\
\hline & 96 & 0.0 & 0.0003 & 0.0050 \\
\hline & 100 & 0.0001 & 0.0018 & 0.0067 \\
\hline \multirow[t]{4}{*}{0.550} & 80 & 0.0014 & 0.0040 & 0.0065 \\
\hline & 96 & 0.0006 & 0.0035 & 0.0067 \\
\hline & 120 & * & - & 0.0046 \\
\hline & 100 & 0.0003 & -0.0002 & 0.0088 \\
\hline \multirow[t]{4}{*}{0.5505} & 80 & 0.0035 & 0.0080 & 0.0088 \\
\hline & 96 & 0.0028 & 0.0060 & 0.0080 \\
\hline & 120 & 0.0025 & 0.0049 & 0.0035 \\
\hline & 100 & 0.0016 & 0.0022 & 0.0084 \\
\hline \multirow[t]{4}{*}{0.551} & 80 & 0.0068 & 0.0128 & 0.0111 \\
\hline & 96 & 0.0068 & 0.0095 & 0.0079 \\
\hline & 120 & 0.0084 & 0.0083 & 0.0068 \\
\hline & 100 & 0.0043 & 0.0133 & 0.0128 \\
\hline \multirow[t]{4}{*}{0.5515} & 80 & 0.0107 & 0.0142 & 0.0119 \\
\hline & 96 & 0.0114 & 0.0104 & 0.0098 \\
\hline & 120 & 0.0133 & 0.0087 & 0.0082 \\
\hline & 100 & 0.0081 & 0.0125 & 0.0119 \\
\hline \multirow[t]{3}{*}{0.552} & 80 & 0.0156 & 0.0150 & 0.0126 \\
\hline & 96 & 0.0147 & 0.0146 & 0.0119 \\
\hline & 100 & 0.0118 & 0.0179 & 0.0143 \\
\hline
\end{tabular}

considerable statistical errors more evidence is ought to be presented, which will be done in sect. 6 .

\section{Thermodynamics on the interface}

The method of investigating the order-disorder kink proposed in refs. $[6,8]$ has the great advantage that the interface under investigation is localized in a preselected plane of the lattice. The centre of the kink is pinned to the location of the sign change of $\Delta \beta$, the shift in the coupling forcing the two halves of the system 
into different phases. The distributions of the energy density and of the order parameter then show the expected qualitative behavior on the two sides of this plane.

As we have shown in constructing fig. 5 , it is possible also in our approach to achieve a localization of the kink in a preselected position by demanding that the plane with minimal magnetization be localized appropriately. The actual value of the minimal magnetization becomes a variable, naturally associated with the tip of the kink:

$$
m_{\min }=\min _{x_{11}}\left(m\left(x_{\|}^{\prime}\right)\right)
$$

With this intuitive interpretation the requirement on the localization can be relaxed. We average $\boldsymbol{m}_{\min }$ over the conventional thermodynamic ensemble and still preserve its correspondence to the (fluctuating) location of the interface. It is an advartage of the present approach that the application of fixed boundary conditions in a large enough volume will not distort the interface (allow for example capillary waves too). Also there is no need for extrapolation in any variable similar to $\Delta \beta$.

Similarly one can define a width variable for the kink making use of the classes $C\left(x_{\min }\right)$ defined at the end of sect. 3. For each class profile (see fig. 5) one measures the distance

$$
i(\beta)=x_{+}(m=\langle M\rangle(\beta))-x_{-}(m=\langle M\rangle(\beta)),
$$

where $x_{+}\left(x_{-}\right)$denotes the location of those planes to the right (left) of the plane with $m_{\min }$ on which the expectation value for the plane magnetization $m\left(x_{\|}\right)$ becomes larger than the bulk average $\langle M\rangle$. [For definiteness we use $\langle M\rangle$ calculated in $\left.V_{\text {eff }}(10)\right]$. An average over all classes in the internal part of the cylinder then yields the mean width for a given $\beta$.

In the mean field description these quantities have a clear geometrical meaning. The complete wetting is realized by $\left\langle m_{\min }\right\rangle$ approaching zero and the width of the order-order interface increasing without any limit. Actually in ref. [13] scaling laws typical for a continuous phase transition were found for them:

$$
\begin{aligned}
\left\langle m_{\min }\right\rangle \sim\left(\beta-\beta_{\mathrm{c}}\right)^{b}, & b_{\mathrm{M} . \mathrm{F} .} \approx 0.40 \pm 0.03, \\
\langle\bar{l}\rangle \sim\left(\beta-\beta_{\mathrm{c}}\right)^{-\psi}, & \psi_{\mathrm{M} . \mathrm{F} .} \approx 0.25 \pm 0.03 .
\end{aligned}
$$

By the above intuitive relationship based on the proposed class decomposition of the samples, the average of $m_{\min }$, defined in eq. (35), and that of $\bar{l}$, defined in eq. (36), were confronted with the scaling laws. In addition, the average magnetic 


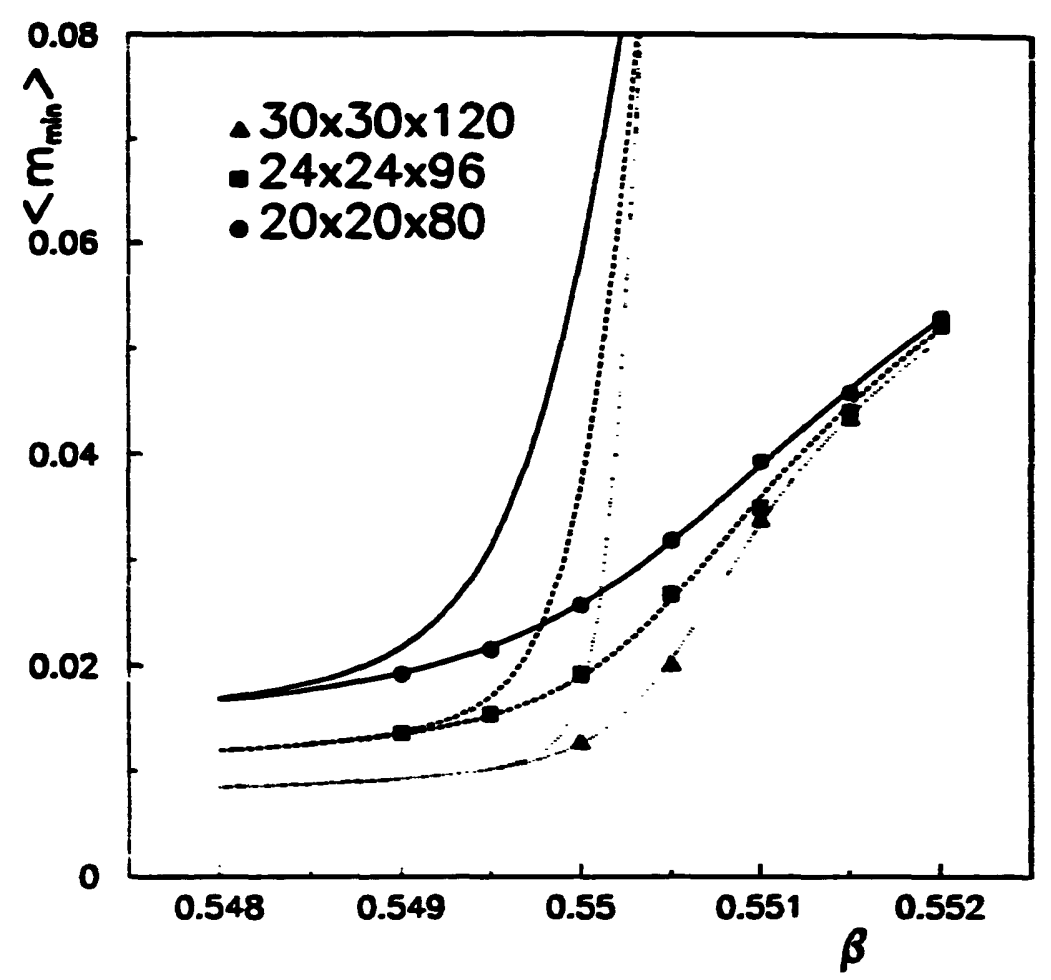

Fig. 8. The average minimal magnetization, $\left\langle m_{\min }\right\rangle$, for par.b.c. (upper branch) and for rot.b.c. (lower branch). Data points are shown only for rot.b.c.; statistical errors are smaller than the size of the symbols.

susceptibility of $m_{\min }$ can be investigated too:

$$
\chi\left(m_{\min }\right)=L_{\perp}^{2}\left(\left\langle m_{\min }^{2}\right\rangle-\left\langle m_{\min }\right\rangle^{2}\right)
$$

In fig. 8 the average of the minimal magnetization measured configuration by configuration is displayed for $V=20^{2} \times 80,24^{2} \times 96$, and $30^{2} \times 120$ both for rot.b.c. and par.b.c. One promptly recognizes that the curves obtained from lattices with par.b.c. break away from zero rather abruptly as $\boldsymbol{\beta}$ approaches the first-order transition point of the three-dimensional Potts model. In case of rot.b.c., however, the sequence of magnetization curves seems to converge smoothly with increasing volume to a limiting function, rising from zero to finite values starting at $\boldsymbol{\beta}_{\mathrm{c}}$. This is characteristic of continuous transitions taking place in the two-dimensional interface.

For incomplete wetting one would expect that in the infinite volume limit the minimal magnetization stays non-zero and the width of the interface stays finite at $\beta_{c}$. A quantitative analysis of the $\beta$-dependence of the width of the interface shown in fig. 5 and of the minimal magnetization (fig. 8), however, strongly supports the occurrence of complete wetting in the three-dimensional three-state Potts model. The scaling law proposed in eq. (37) has been examined by varying $\boldsymbol{\beta}_{\mathrm{c}}$ 


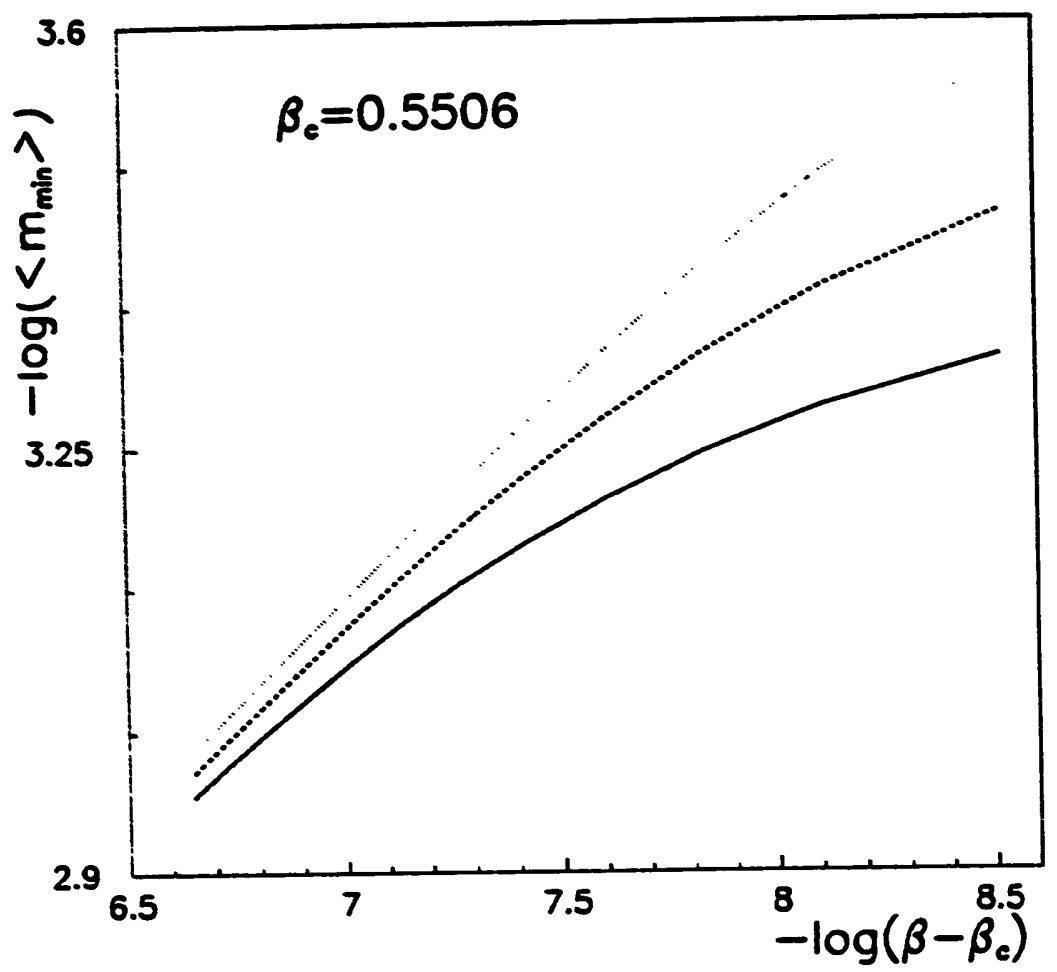

Fig. 9. $\left\langle m_{\min }\right\rangle$ versus $\beta-\beta_{c}$ on the $\log -\log$ scale for testing the scaling law in eq. (37).

in the range indicated by the bulk characteristics (see sect. 2$): \beta \in(0.5504-0.5506)$. It is obvious from fig. 9 that for smaller volumes, considerable deviations are observed from the straight-line behavior on the logarithmic scale. The largest volume, however, allows a convincing estimate of the magnetic exponent, which appears to be compatible with the mean-field value [13]

$$
b=0.35 \pm 0.05
$$

The error expresses the variation of the slope due to the uncertainty in the determination of $\boldsymbol{\beta}_{\mathrm{c}}$.

From the magnetization profile shown in fig. 5 for a single class of configurations (those with $m_{\min }$ being located at $x_{\|}=L_{\|} / 2$ ) a tendency for widening of the interface as $\beta$ approaches $\beta_{c}$ is obvious. This is true also for all other profile classes with $m_{\min }$ not too close to the boundaries. We used an average over the $\mathbf{1 0}$ innermost classes in order to extract the width of the interface at $\beta=0.551,0.5515$ and 0.552 . The exponent was then found from a fit with eq. (38):

$$
\psi=0.21 \pm 0.03
$$

This again turns out to be compatible with the mean-field behavior. 
These observations give support to a continuous two-dimensional critical phenomenon occurring simultaneously with the bulk discontinuous one. Indeed, we have also observed a maximum in the interface susceptibility, eq. (39), at $\beta \approx 0.5514$ $\left(V=80 \times 20^{2}\right)$ which moves to $\beta \approx 0.5509\left(V=30^{2} \times 120\right)$. However, the rather modest transverse size prevents a fully trustable statement on the behavior of the susceptibility.

\section{Conclusions}

In this paper we have presented a detailed study of the order-order interface in the three-dimensional three-state Potts model in cylindrical geometries with fixed boundary variables at its ends. The main results are the following:

(i) The finite-size effects on bulk quantities are similar in periodic systems and in the inner volume of the cylinder when the boundary spins are parallel on the opposite ends. With rotated boundary conditions the crossover between the highand low- $\beta$ regimes is much smoother on the moderate size lattices studied by us.

(ii) An effective one-dimensional three-state model is associated rather naturally to the description of the interface. With the help of this mapping, we presented evidence that a single interface predominates already for $\beta \approx \beta_{c}$ when rotated boundary conditions are applied.

(iii) The selection of the plane where the plane-averaged magnetization reaches its minimum is a rather successful way to localize the interface. The magnetization and the magnetic susceptibility in this plane seem to indicate a continuous phase transition, whose magnetic and width exponents,

$$
b=0.35 \pm 0.05 \text { and } \psi=0.21 \pm 0.03
$$

were found compatible with mean-field estimations. This verifies the result of Lipowsky that the upper critical dimension for wetting phenomena is three [21].

(iv) The surface tension and the action asymmetry appear to be two independent quantitative signals for the presence of the interface. Exploiting the physically enlightening FS-interpolation algorithm, reliable estimates of the surface tension can be obtained by comparing samples with rot.b.c. and par.b.c.:

$$
\alpha_{\text {ord }} \geqslant 0.0048
$$

Two independent methods were applied to the action asymmetry, leading to results that are consistent with each other:

$$
\mathscr{A}_{\text {ord }}=0.0078 \pm 0.0015
$$

(v) A comparison between the magnitude of the action asymmetry induced by order-order interfaces and previous results [eq. (30)] for order-disorder inter- 
faces, as well as the surface criticality indicated by the behavior of the minimal magnetization, provides strong evidence for complete wetting taking place at $\boldsymbol{\beta}_{\mathrm{c}}$ in the three-dimensional three-state Potts model.

Mapping the system on an effective one-dimensional three-state model for the description of the interface and looking into the thermodynamics of the fluctuating plane of minimal magnetization in systems of larger cross section merit further investigation.

The authors are indebtcd to J. Engels providing them with an optimized Ferrenberg-Swendsen program for the data analysis and to B. Pendleton for his comments on the manuscript. The numerical investigations have been performed on the Cray X-MP at CERN.

\section{References}

[1] G.M. Fuller, G.J. Mathews and C.R. Alcock, Phys. Rev. D37 (1988) 1380

[2] R.A. Malanay and W.A. Fowler, Astrophys. J. 333 (1988) 14

[3] H. Kurki-Suonio, R.A. Matzner, K.A. Olive and D.N. Schramm, Astrophys. J. 353 (1990) 406

[4] K. Kajantie, Helsinki preprint HU-TFT-89-31 (1989)

[5] C. Greiner, D.H. Rischke, H. Stöcker and P. Koch, Phys. Rev. D38 (1988) 2797

[6] K. Kajantie and L. Kärkkäinen, Phys. Lett. B214 (1988) 595

[7] K. Kajantie, L. Kärkkäinen and K. Rummukainen, Nucl. Phys. B333 (1990) 100

[8] S. Huang, J. Potvin, C. Rebbi and S. Sanielevici, Boston preprint BUHEP-89-34, January 1990

[9] J. Potvin and C. Rebbi, Phys. Rev. Lett. 62 (1989) 3062

[10] K. Kajantie, L. Kärkkäinen and K. Rummukainen, Phys. Lett. B223 (1989) 213

[11] J. Potvin and C. Rebbi, Nucl. Phys. B (Proc. Suppl.) 17 (1990) 223

[12] Z. Frei and A. Patkós, Phys. Lett. B222 (1989) 469

[13] Z. Frei and A. Patkós, Phys. Lett. B229 (1989) 102

[14] B. Svetitsky and L.G. Yaffe, Phys. Rev. D26 (1982) 963; Nucl. Phys. B210 [FS6] (1982) 423

[15] M. Fukugita, A. Ukawa and M. Okawa, Nucl. Phys. B337 (1990) 181

[16] R.V. Gavai, F. Karsch and B. Petersson, Nucl. Phys. B322 (1989) 738

[17] M. Fukugita, M. Mino, A. Ukawa and M. Okawa, Nucl. Phys. B (Proc. Suppl.) 17 (1990) 180

[18] F. Karsch, Simulating the quark-gluon plasma on the lattice, in: Quark-gluon plasma-Advanced series on directions in high energy physics, Vol. 6, ed. R.C. Hwa (World Scientific, Singapore, 1990)

[19] K. Rummukainen, Z. Phys. C47 (1990) 157

[20] R. Lipowsky, Critical behaviour of interfaces: wetting, surface melting and related phenomena, Jül-Spez-438 (1988)

[21] R. Lipowsky, Phys. Rev. Lett. 52 (1984) 1429

[22] S. Dietrich, Wetting phenomena, in: Phase transitions and critical phenomena, Vol. 12, ed. C. Domb and J. Lebowitz (Academic Press, New York, 1988) p. 10

[23] A.M. Ferrenberg and R.H. Swendsen, Phys. Rev. Lett. 63 (1989) 1195

[24] M.S.S. Challa, D.P. Landau and K. Binder, Phys. Rev. B34 (1986) 1814

[25] M.E. Fisher and A.N. Berker, Phys. Rev. B26 (1982) 2507 